\title{
Physical activity in an air-polluted environment: behavioral, psychological and neuroimaging protocol for a prospective cohort study (Healthy Aging in Industrial Environment study - Program 4)
}

S. Elavsky ${ }^{1,2^{*}}$ (D, V. Jandačková1,3, L. Knapová1,2, V. Vašendová', M. Sebera', B. Kaštovská', D. Blaschová1, J. Kühnová4, R. Cimler ${ }^{4}$ D. Vilímek ${ }^{1,5}$, T. Bosek ${ }^{1}$, J. Koenig ${ }^{6,7}$ and D. Jandačka ${ }^{1}$

\begin{abstract}
Background: Air pollution has been linked to increased mortality and morbidity. The Program 4 of the Healthy Aging in Industrial Environment study investigates whether the health and wellbeing benefits of physical activity (PA) can be fully realized in individuals living in highly polluted environments. Herein, we introduce the behavioral, psychological and neuroimaging protocol of the study.

Methods: This is a prospective cohort study of $N=1500$ individuals aged 18-65 years comparing: (1) individuals living in the highly polluted, industrial region surrounding the city of Ostrava $(n=750)$, and (2) controls from the comparison region with relative low pollution levels in Southern Bohemia $(n=750)$. Quota sampling is used to obtain samples balanced on age, gender, PA status (60\% active runners vs. 40\% insufficiently active). Participants are screened and complete baseline assessments through online questionnaires and in-person lab-based assessments of physiological, biomechanical, neuroimaging and cognitive function parameters. Prospective 12-month intensive monitoring of air pollution and behavioral parameters (PA, inactivity, and sleep) follows, with a focus on PA-related injuries and psychological factors through fitness trackers, smartphones, and mobile apps. Subsequently, there will be a 5-year follow-up of the study cohort.

(Continued on next page)
\end{abstract}

\footnotetext{
* Correspondence: steriani.elavsky@osu.cz

${ }^{1}$ Faculty of Education, University of Ostrava, Ostrava, Czech Republic

${ }^{2}$ Faculty of Informatics, Masaryk University, Brno, Czech Republic

Full list of author information is available at the end of the article
}

(C) The Author(s). 2021 Open Access This article is licensed under a Creative Commons Attribution 4.0 International License, which permits use, sharing, adaptation, distribution and reproduction in any medium or format, as long as you give appropriate credit to the original author(s) and the source, provide a link to the Creative Commons licence, and indicate if changes were made. The images or other third party material in this article are included in the article's Creative Commons licence, unless indicated otherwise in a credit line to the material. If material is not included in the article's Creative Commons licence and your intended use is not permitted by statutory regulation or exceeds the permitted use, you will need to obtain permission directly from the copyright holder. To view a copy of this licence, visit http://creativecommons.org/licenses/by/4.0/ The Creative Commons Public Domain Dedication waiver (http://creativecommons.org/publicdomain/zero/1.0/) applies to the data made available in this article, unless otherwise stated in a credit line to the data. 


\begin{abstract}
(Continued from previous page)
Discussion: The design of the study will allow for (1) the assessment of both short-term variation and long-term change in behavioral parameters, (2) evaluation of the incidence of musculoskeletal injuries and psychological factors impacting behavior and injury recovery, and (3) the impact that air pollution status (and change) has on behavior, psychological resilience, and injury recovery. Furthermore, the integration of MRI techniques and cognitive assessment in combination with data on behavioral, biological and environmental variables will provide an opportunity to examine brain structure and cognitive function in relation to health behavior and air pollution, as well as other factors affecting resilience against and vulnerability to adverse changes in brain structure and cognitive aging. This study will help inform individuals about personal risk factors and decision-makers about the impact of environmental factors on negative health outcomes and potential underlying biological, behavioral and psychological mechanisms. Challenges and opportunities stemming from the timing of the study that coincided with the COVID-19 pandemic are also discussed.
\end{abstract}

Keywords: Air pollution, Environment, Physical activity, Health, Cognition, Neuroimaging, Aging

\section{Background}

\section{Air pollution and health}

Life expectancy has been increasing globally with the number of people aged 65 years and above expected to more than double in the next several decades. The projected increase in Europe and North America, specifically, is $48 \%$ by the year 2050 [1]. These important gains in life expectancy are outweighed by increasing trends in comorbidity. One important contributing factor to this trend is air pollution. According to the World Health Organization (WHO), 4.2 million deaths worldwide every year are attributed to ambient air pollution, with $91 \%$ of the world's population living in places exceeding WHO air quality guidelines [2, 3].

Air pollution has both acute and chronic effects on human health. Exposure to air pollution has been related with premature mortality and reduced life expectancy $[4,5]$. Of the premature deaths estimated to be caused by ambient air pollution, up to $72 \%$ occur from ischemic heart disease and stroke, $8 \%$ from chronic obstructive pulmonary disease, $14 \%$ from acute lower respiratory tract infection, and $6 \%$ are related to lung cancers [6]. Interestingly, a recent study examining the relationship between air-pollution and coronavirus fatality concluded that exposure to air pollution may be an important contributor to death related to COVID-19; almost $80 \%$ of deaths were observed in 5 regions located in north Italy and central Spain, that also had the highest NO2 concentrations $[7,8]$. The negative effects of air pollution have been demonstrated across a range of pollution parameters (e.g., particulate matter - $\mathrm{PM}_{10}, \mathrm{PM}_{2.5}$, nitrogen dioxide $\mathrm{NO}_{2}$, carbon monoxide - $\mathrm{CO}$, ozone, sulphur dioxide - $\mathrm{SO}_{2}$, or benzo [a]pyrene) and are thought to occur through a range of physiological mechanisms involving inflammation, oxidative damage, lipid peroxidation, carcinogenic and genotoxic effects [4, 9-11]. Air pollution can however also impact health indirectly through its effect on human behavior.

\section{Air pollution and behavior}

One behavioral factor directly linked to both - exposure to air pollution and health outcomes -is physical activity.
Physical activity (PA) brings about many health benefits, however, when performed outdoors in polluted environments, it can increase exposure to air pollutants [11-13]. Additionally, avoidance of PA due to poor air quality may further undermine health or exacerbate disease. An et al. [14] conducted a systematic review and meta-analysis of studies examining the links between air pollution and physical activity. Across seven studies there was a negative association between PA and air pollution $\left(\mathrm{PM}_{2.5}\right.$ or $\left.\mathrm{PM}_{10}\right)$ and a positive association was found with inactivity. The results suggested that at least cross-sectionally, air pollution is associated with reduced physical activity.

Data from the Behavior Risk Factors Surveillance Survey (BRFSS) in the US also indicated that the level of inactivity during leisure time correlates positively with ambient $\mathrm{PM}_{2.5}$ [15]. In another study of mobile app users in China, people reduced the frequency of outdoor exercise at an increasing degree of air pollution, although there were no differences in average distance and duration of exercise once exercise was initiated [16]. Results of this Chinese study are consistent with a study by Roberts et al. [17], where increased air pollution $\left(\mathrm{PM}_{2.5}\right)$ was linked with increased odds of physical inactivity in the US adult population. Finally, Yu et al. [18] found a positive association between sedentary behavior (number of total weekly hours of sitting) and air quality index (AQI), $\mathrm{PM}_{2.5}, \mathrm{PM}_{10}, \mathrm{NO}_{2}$ parameters among Tsinghua University in Beijing students. Altogether, these studies suggest that air pollution shows a negative association with PA and a positive association with inactivity or sedentary behavior, although longitudinal investigations are scarce.

Among other behaviors linked to the exposure to air pollutants is sleep. In a representative study of Chinese children aged 2-17 years, it has been shown that exposure to air pollutants increases the odds for sleep disorders and sleep-disorder symptoms (with strongest association for $\mathrm{PM}_{1}$ and $\mathrm{PM}_{2.5}$ ) [19]. In a study of college freshmen across a five-year period, increasing concentrations in $\mathrm{AQI}, \mathrm{PM}_{2.5}, \mathrm{PM}_{10}$, and $\mathrm{NO}_{2}$ were also 
associated with a reduction in daily hours of sleep [20]. As a behavior, sleep has been linked to many health outcomes, it impacts wellbeing and is also associated with physical activity. Yet there appear to be few studies on the interplay between sleep, physical (in) activity and air pollution [21, 22]. Insufficient moderate PA and sleep duration may also contribute to altered behavioral responses to stress and the development of depressive symptoms [23], underscoring their relevance for mental health and the need to consider links between these behaviors, air pollution and mental health.

\section{Air pollution and mental health}

Air pollutants may play a role in the etiology of psychiatric disorders including depression, anxiety disorders, suicidal behavior and psychoses due to their toxicity on the central nervous system [24-26]. For example, exposure to $\mathrm{PM}_{2.5}$ has been associated with an increased risk of depressive symptoms in older women without prior history of depression or cognitive impairment [27] and with both depressive and anxiety symptoms in community-dwelling older adults [28]. Additionally, increases in $\mathrm{PM}_{10}, \mathrm{NO}_{2}$, and $\mathrm{O}_{3}$ have been linked to depressive symptoms in the elderly [29]. In a longitudinal, nationally representative study, annual-average measures of $\mathrm{PM}_{2.5}$ were significantly associated with increased psychological distress across a 12-year period (after controlling for other sociodemographic and health-related factors) [30]. Yet, a study involving four European cohorts (the Netherlands, Germany, Norway, Finland) and a total of $N=70,928$ participants failed to find consistent patterns of association between depressed mood and air pollutants across the four cohorts [31], setting off a debate concerning the strength and consistency of the association and the heterogeneous methods used to study it [32, 33]. Intriguing were nonetheless the results of a small study that utilized experience sampling methods and revealed associations between a real-time objective air pollution index (derived from hourly real-time data released by the Beijing Environmental Protection Bureau) and in-time ratings of subjective wellbeing (negative association with affective ratings) and eudaimonic wellbeing (positive association with ratings of meaning and purpose in life) [34]. Both physical (in) activity and sleep covaried with ratings of psychological wellbeing and distress, and both behaviors were linked with risk of mental health disorders such as depression [35-39]. To what extent this "protection" is maintained when PA is performed in air polluted environments requires further study. Especially studies with objective measures of physical (in) activity and/or sleep and those allowing for both long-term and acute (real-time) capture of air polluting parameters on ratings of wellbeing and mental health outcomes are urgently needed [10].

\section{Air pollution and neurocognitive health}

There is a growing body of evidence suggesting that exposure to air pollution might have neurotoxic effects that culminate over time to neuronal damage and loss, and changes in brain structures and function at all ages [40], leading to altered neurodevelopment and cognitive dysfunction, an important intermediate event in the pathogenesis of dementia [41]. Epidemiological studies have shown that air pollution is associated with decreased cognitive performance, neuroimaging correlates and increased risk of cognitive impairment and dementias [42-46]. For example, in a recent study in $n=998$ older women aged over 73 years long-term $\mathrm{PM}_{2.5}$ exposure was associated with a greater decline in immediate recall and learning (but not with other scores of memory), and with progressive atrophy of grey matter, indicative of increased neuroanatomic risk for Alzheimer's disease [45]. A meta-analysis of four cohort studies from Canada, Taiwan, the UK and the US [47] including more than 12 million elderly aged 50 years and older reported a 3-fold increase in dementia risk with chronic exposure to $\mathrm{PM}_{2.5}$ (HR 3.26, 95\% CI $1.20-5.31$ per $10 \mu \mathrm{g} / \mathrm{m} 3$ ). Among older US adults, long-term exposure to higher levels of air-pollutants (i.e. $\mathrm{PM}_{2.5}, \mathrm{PM}_{10}$ and $\mathrm{NO}_{2}$ ), were cross-sectionally and longitudinally related to pronounced cognitive decline including memory, executive function and language domains [48]. Furthermore, increasing exposure to $\mathrm{PM}_{2.5}$ was associated with lower verbal learning, exposure to $\mathrm{NO}_{2}$ with lower logical memory, and $\mathrm{O}_{3}$ exposure with lower executive function in a study of cognitively intact middle-aged and older US adults [49]. In the Nurses' Health Study, higher longterm concentrations of both $\mathrm{PM}_{2.5-10}$ and $\mathrm{PM}_{2.5}$ were linked to a significantly faster cognitive decline over a two-year period [50]. Studies integrating environmental epidemiology and neuroimaging suggest that white matter, cortical grey matter and the basal ganglia could be targets of air-pollution and that these alterations on the brain-level might underlie the association between airpollution and cognitive dysfunction in humans, however, the results are inconsistent possibly due to considerable heterogeneity across study populations, MRI methods, pollutants, and methods of cognitive investigation $[46,51]$. The exact mechanisms by which air pollutants cause damage to the central nervous system (CNS) are unknown. Several biological mechanisms have been hypothesized with strongest evidence surrounding pathways of systemic inflammation and oxidative stress [52]. Physical activity, on the other hand, has been shown to be protective for brain and cognition and effective in preventing or delaying the onset of cognitive impairment [53]. Regular PA is thought to improve cognitive function [54] and increase neural plasticity through modifications in vascular physiology, reduction of neuroinflammation and cerebral 
oxidative stress, and through increases of serum brainderived neurotrophic factor (BDNF) induced by exercise $[55,56]$. There is some evidence suggesting that regular exercise in highly polluted air might not result in the same psychological and neurological benefits that are observed in non-polluted environments [57]. However, whether regular exercise in highly polluted air may actually negatively impact cognition as well as brain structure and function, remains unresolved. Importantly, the optimal level of regular PA that is beneficial from a neurological perspective and mental health may differ depending on air quality.

\section{The present study}

Unfortunately, the interpretation of existing findings linking air pollution to mental or neurocognitive health remains limited given the majority of retrospective study design, varying observational periods and methods, and failure to account for confounding factors such as age or health status [58]. Indeed, the strongest associations are often found in individuals with multiple risk factors (e.g., lower socio-economic status (SES), poorer health status or a history of depression) $[28,59]$. Other limitations of studies targeting behavioral outcomes such as PA stem from the fact that they mostly rely on self-reports, retrospective assessments of behavior, which can be biased, and collect averaged annual data on air quality, thereby lacking the dynamic sensitivity to detect acute/shortterm changes while also evaluating long-term trends. Important technological advances have taken place in the last several decades, allowing for near real-time assessments of both behavior and psychological outcomes. With smartphone use becoming ubiquitous [60] and the availability of commercial wearable technologies ever increasing, there has never been a more opportune time to make use of these tools to assess the interplay between behavior, psychological states and air quality parameters. Combined with robust laboratory-based methods for the comprehensive assessment of health status of individuals, these methods, sometimes referred to as Dynamic Real-time Ecological Ambulatory Methodologies (DREA M) [61], may help to significantly improve the quality of assessment protocols in prospective studies, addressing links between air-pollution, health, and wellbeing.

Herein, we describe the behavioral, psychological, and neuroimaging aspects of the study protocol from Program 4 of the Healthy Aging in Industrial Environment (HAIE) project, including how mobile technology is utilized to collect real-time data on air quality, behavior, and daily psychological functioning. HAIE consists of four research programs that are interlinked using the same selected population groups study the impact of individual and environmental determinants of health, wellbeing and healthy aging. Program 4 of the HAIE project
(4HAIE) specifically focuses on the links between air pollution, biomechanical, physiological, psychosocial, and sociodemographic variables (and their interaction) on the incidence of running-related injuries, physical (in) activity, health and quality of life. Herein, we describe the protocol for the assessment of behavioral, psychosocial and neurocognitive outcomes as part of the study, including key domains of interest in the relationship between air pollution, selected behavioral parameters, psychological functioning and neuroimaging correlates.

\section{Methods}

\section{Study sample and recruitment}

The primary objective of the 4HAIE project is to establish prospective study cohorts to assess the effects of air pollution on health and quality of life across the lifespan. The cohorts are recruited from two different regions within the Czech Republic that differ in air quality. The highly polluted Moravian-Silesian Region (MSR) of the Czech Republic (where life expectancy is about 2 years lower compared to the Czech Republic average [62] and the control South Bohemian Region (SBR) with comparatively low levels of air pollution (See Fig. 1). The Moravian-Silesian region has been long term European hotspot of ambient benzo [a] pyrene and particulate matter pollutants [63]. Exposure to benzo [a] pyrene air pollution in MSR (Ostrava-Radvanice) has been the highest in the European union for a decades [64].

For the 4HAIE study, specifically, the recruitment of study participants was commissioned to a professional social science research and marketing company selected through a publicly advertised tender. The sample is collected using quota sampling based on location, age, gender, and PA status (active-runner vs. inactive) (for details see Table 1 ).

A total of $N=1500$ participants aged $18-65$ years will be recruited for this study. Of these, $n=900(60 \%)$ will be physically active runners and $n=600$ inactive controls. The runners and inactive controls will be split evenly between two regions: the experimental highlypolluted industrial region (Moravian-Silesian Region with the exception of the district of Bruntal and selected counties in the district of Frydek Mistek) and the control low-pollution level region (South Bohemian Region). The selected sample size was determined as the largest sample size feasible, allowing for stratification of the cohort by age group and analysis of outcomes targeted by the interdisciplinary teams involved. No formal unifying power analysis was however conducted.

Participants are being recruited from the community using various approaches including in-person recruitment through agency workers, online (using social media, job portals and other websites) and offline 


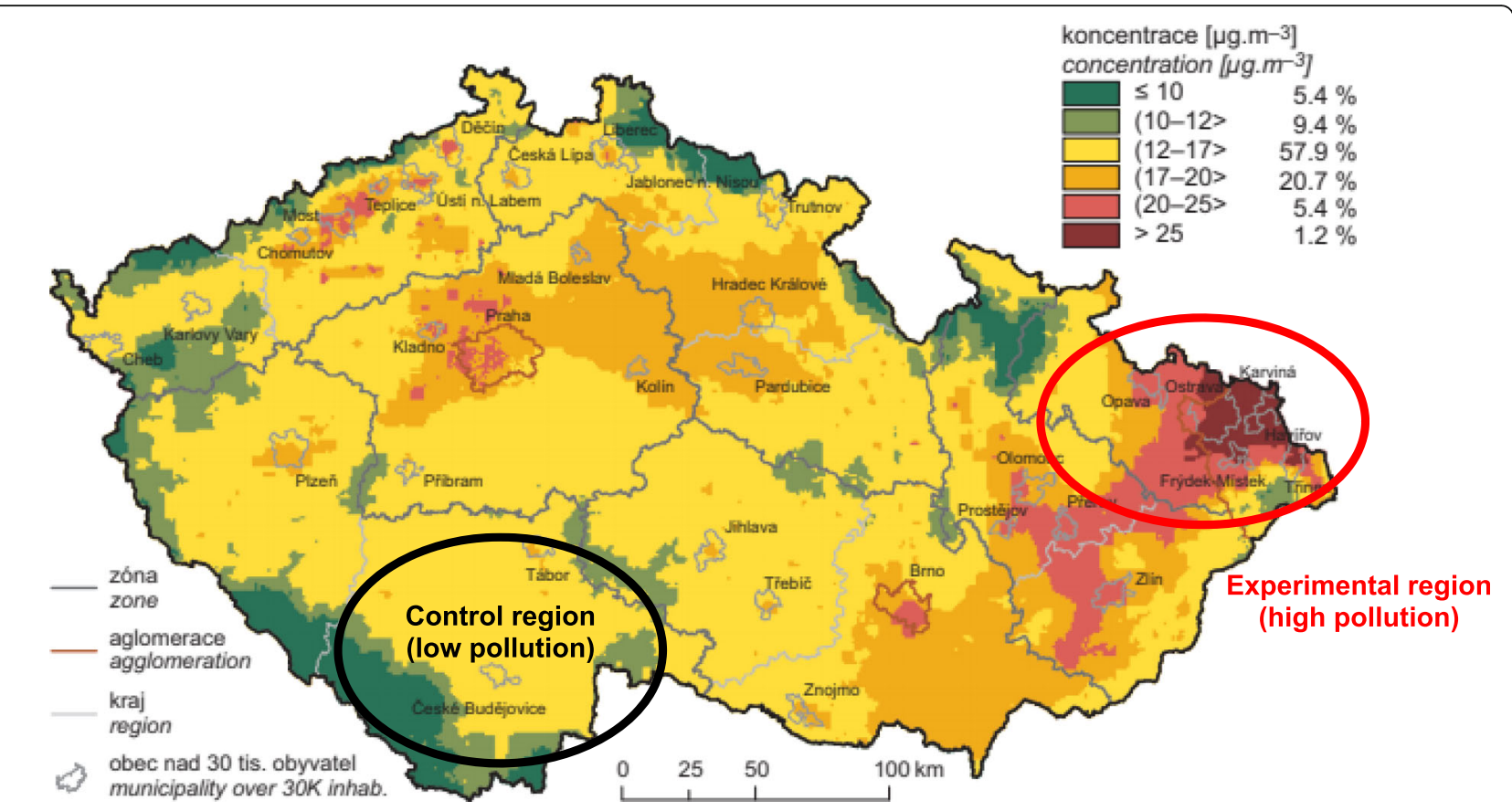

Note. Figure adapted with permission from the Czech Information System on Air Quality (ČHMÚ). Data are from 2018 and reflecting limit value valid from 2020.

Fig. 1 Field of annual average concentration of PM2.5 in 2018 indicating limit value valid from 2020. The figure was adapted from publicly available source with author permission (Air Quality Information System. Air Pollution in the Czech Republic in the year 2018. 2018. https://www. chmi.cz/files/portal/docs/uoco/isko/grafroc/18groc/gr18cz/Prilohall_CHMU2018.pdf. Accessed 22 Jun 2020)

advertisements (billboards, newspaper ads, flyers). Recruitment activities focused specifically on runners have been conducted, such as presentations and information booths at running races, through running clubs, and at other relevant community events. Participant recruitment began in February 2019, with the first participants undergoing baseline testing in April 2019. To date (November 2020), $n=1012$ participants have been recruited and underwent baseline assessments, of which $n=360$ completed the entire 12-month study. Baseline testing was expected to be completed by the end of 2020, with

Table 1 Recruitment quotas based on age, region (SBR = South Bohemian Region, MSR = Moravian-Silesian Region) and activity status

\begin{tabular}{llllll}
\hline \multirow{2}{*}{$\begin{array}{l}\text { Age } \\
\text { group }\end{array}$} & \multicolumn{2}{l}{ Runners (60\%) } & & \multicolumn{2}{l}{ Inactive Controls (40\%) } \\
\cline { 2 - 3 } & SBR & MSR & & SBR & MSR \\
\hline $18-25$ & 90 & 90 & & 60 & 60 \\
$>25-35$ & 90 & 90 & & 60 & 60 \\
$>35-45$ & 90 & 90 & & 60 & 60 \\
$>45-55$ & 90 & 90 & & 60 & 60 \\
$>55-65$ & 90 & 90 & & 60 & 60 \\
Total N & 450 & 450 & & 300 & 300 \\
\hline
\end{tabular}

12-month intensive monitoring of all participants concluding by the end of 2021. Due to restrictions imposed in the Czech Republic as part of combating COVID-19, testing was interrupted between March 13-April 30, 2020, extending the anticipated recruitment and baseline testing period until June 2021. Up-to-date status of recruitment progress (along with the sociodemographic composition of the sample) can be followed on the study website https://www.4haie.cz/.

\section{Participant incentives}

Participants receive small financial compensation for the baseline laboratory assessments of 40EUR for MSR participants and 70EUR for SBR participants (to compensate for the additional travel time from SBR). Free transportation is provided for SBR participants. Upon completion of the baseline testing, participants also receive results from several baseline assessments (e.g., graded-exercise test, body composition) and a gift bag. Throughout the 12-month monitoring period all participants are eligible for a lottery to win one 185EUR gift card every month. The number of entries into the lottery is based on completing different aspects of the study and reflects the participant's compliance in the given month. 
Upon study completion the participants receive detailed personalized feedback on their behavior, survey responses and air pollution data in the form of a final study report.

\section{Inclusion and exclusion criteria}

All participants had to be non-smokers between the ages of 18-65 years at the start of the study. Active runners had to meet public PA guidelines (150 min/week moderate or $75 \mathrm{mins}$ /week vigorous PA or an equivalent combination of the two as per WHO recommendations; [65]), be running at least $10 \mathrm{~km}$ per week in the last 6 weeks and plan to continue running for the next 12 months. Inactive controls do not meet the public PA recommendations but are capable of normal PA including running (i.e. no physician diagnosed restrictions). Both runners and inactive participants must live in the region for the last 5 years and should have no plans to move outside the region in the next 12 months. All participants are required to own a smartphone (with Android $\geq 5.0$ or iOS) and have internet access (through WiFi or mobile data).

Main exclusion criteria include being a smoker, having an acute health problem (e.g., illness) or injury/surgery/ pain preventing normal PA (within the last 6 weeks) and having contraindications to magnetic resonance imaging (MRI) or dual-energy x-ray absorptiometry (DXA) (e.g., pregnancy, radiological examination in the last 7 days using iodine / barium contrast agents, pacemaker, radioactive body, surgical staples, insulin pump, cochlear implant, other metal implants and foreign bodies such as shrapnel, etc.).

\section{Study timeline and procedures}

Interested participants complete screening online and by telephone. Eligible participants are then invited to complete first two baseline questionnaires from home (through the Qualtrics online survey platform) and are scheduled for a 2-day laboratory assessment. Upon arrival to the laboratory (up to 4 participants are tested each day), informed consent is administered in person and participants obtain a hard copy of the signed informed consent to keep for their records. The 2-day laboratory assessments include a series of physiological, biomechanical, anthropometric, cognitive and MRI assessments (also described in other protocols currently under review). Participants also complete two additional online questionnaires while in the laboratory and undergo in-person training in study procedures. During this training, lab staff install mobile apps (a custommade survey app and the Fitbit fitness app) on the participants' smartphones. Participants are familiarized with the study protocol, the apps, and receive detailed instructions for using a fitness bracelet (Fitbit Charge 3).
The orientation to the study procedures, apps, and fitness bracelet takes approximately $40 \mathrm{~min}$. Cognitive testing and neuroimaging take place in the morning after breakfast on day 2. Cognitive assessment is conducted in a quiet room and takes approximately $45 \mathrm{~min}$; neuroimaging takes about $10 \mathrm{~min}$. The study procedures have been approved by the Ethical Committee of the University of Ostrava.

\section{Questionnaires}

As part of the study, participants complete a series of four separate baseline questionnaires: two at home prior to arrival to the laboratory (Socioeconomic and Psychological Survey) and two sets of questionnaires (Physical Activity Survey and Biomechanical Survey) in the laboratory.

The Socioeconomic Survey, prepared in collaboration with the study partner of Program 1 of the HAIE project (Faculty of Medicine at the University of Ostrava), consists of questions about sociodemographic factors, basic lifestyle factors, risk perception, health status, and quality of life. The completion of the survey takes approximately $40 \mathrm{~min}$. The Psychological Survey includes questions concerning psychological protective factors (such as social support) and risk factors (such as perceived stress, depressive symptoms, or neuroticism). Questions about sleep are also included. The completion of the survey takes approximately $30 \mathrm{~min}$. The Physical Activity Survey completed in the laboratory is composed of questions about physical activity, sedentary behavior, motivational and psychological factors associated physical (in) activity and running history. The completion of the survey takes approximately $45 \mathrm{~min}$. In the Biomechanical Survey $(20 \mathrm{~min})$ participants are asked about injury history and pain. A detailed summary of the questionnaires and measures used in this study can be located on the study website https://www.4haie.cz/data/.

At month 6 and month 12, participants are asked to complete a subset of selected measures online from home again. The completion of each of the follow-up surveys takes approximately $45 \mathrm{~min}$.

\section{Hair samples}

Until a few years ago, cortisol has been analyzed only from blood, saliva or urine to assess acute or short-term circulating cortisol concentrations [66]. Whereas these acute and transient cortisol reactivity are part of the adaptive response to challenge and generally unharmful [67], long-term secretion of the cortisol is considered to play a crucial role in mediating the link between chronic stress and negative effects on physical and mental health [68]. Given the possible negative health effects of chronic long-term stress, in this study we use a new non-invasive, easily conducted and well tolerated 
method of analysis of cortisol from hair samples. Three$\mathrm{cm}$ segment of hair is collected by a trained person with small scissors as close to scalp as possible, according to a validated protocol [69]. Hair samples are taken from the posterior vertex region in accordance with guidelines published by the Society of Hair Testing [70]. Considering that hair grows approximately $1 \mathrm{~cm}$ per month [71], hair samples are obtained in order to evaluate hair cortisol levels representative of the last 3 months [72]. Each sample is wrapped in aluminum foil to protect it from light and humidity and stored in closed plastic bags (marked with the ID, date and initials of laborant) at room temperature. Later, the samples will be analyzed at the Dresden University of Technology, Germany using an Enzyme-Linked Immunosorbent Assay (ELISA), a reliable and valid method to assess hair cortisol levels, which is highly positively correlated with liquid chromatograph-mass spectrometry (LC-MS/MS) [73].

\section{Cognitive assessment}

The neurocognitive session includes five tests assessing memory, language and executive function:

\section{Rey auditory verbal learning test (RAVLT) [74]}

RAVLT is a powerful neuropsychological tool that is used for assessing episodic memory by providing scores of evaluating different aspects of memory including immediate recall, short-term and long-term memory, and verbal learning. The participant is required to learn a list of 15 words presented verbally at 2-s intervals over the course of 5 trials and recall verbally as many of the words as possible in any order after each trial. After approximately $30 \mathrm{~min}$ participants are asked to recall the words without repeated presentation to assess the long-term memory. The RAVLT is sensitive to verbal memory deficits caused by variety of neurological conditions [75].

\section{Verbal fluency test [76]}

We use 2 measures of verbal fluency, phonemic and semantic. Participants are asked to recall verbally as many words beginning with $\mathrm{S}, \mathrm{K}$ and $\mathrm{P}$ (phonemic fluency) and as many animal names (semantic fluency) as they can. Sixty seconds is allowed for each of the 4 tests. Verbal fluency is used to investigate the presence of cognitive impairment, neurodegenerative and psychiatric conditions [77].

Three computerized tests from NIH-Examiner battery developed to reliably and validly assess domains of executive function [78]: Flanker Attention test, Set Shifting task, and Nback and 2Nback tests. Tasks are applicable to a broad range of individuals from different age and ethnic groups. Flanker task is a response inhibition task used to assess the ability to suppress responses that are inappropriate in a specific context; Set shifting task assesses cognitive flexibility and Nback and 2Nback assess working memory. During these tasks frontal brain structures are thought to be activated [78]. We used back translation method to translate the tests into the Czech language. Each participant completes these 3 tasks on laptops in the Psychopy program (version 1.73) [79].

\section{Neuroimaging}

Scanning is carried out at the Department of Human Movement Studies, University of Ostrava, using a $1.5 \mathrm{~T}$ Siemens Magnetom Sempra Scanner with a 16- channel head coil (1.5 T Siemens Magnetom Sempra Scanner; Siemens, Erlanger, Germany). The neuroimaging protocol comprises structural sequences only, lasting approximately $10 \mathrm{~min}$. MRI sequences include high-resolution T1-weighted, T2-weighted and 3-scan trace diffusionweighted imaging. A full description of the MRI parameters adopted in our sequences is provided in Table 2 . Cortical surface reconstruction and subcortical segmentation is completed via FreeSurfer (version 6.2), including total grey and white matter as well as subcortical volumes, cortical thickness and cortical surface area estimates for cortical regions [80]. Images undergo quality control prior to processing by 3 experts: a radiologist with 10 years of experience and two image analysts, each with 2 years of experience. Brain tissues and subcortical regions are visually inspected to ensure an accurate segmentation, gauge the severity of motion, intensity homogeneity, white matter underestimation, pial overestimation and artifacts [80]. Quality control of FreeSurfer reconstructions is based on MELD Protocol 3 [81].

\section{Long-term monitoring and EMA protocol}

Following baseline assessments, participants are monitored for 12 months using the Fitbit Charge 3 monitor. Additionally, participants complete brief surveys administered on their smartphones through a mobile app developed for the study. The intensive assessment incorporates elements of ecological momentary assessment (EMA) and will be administered four times for 2 weeks across the 12-month period (at baseline, month 4, month 8, and month 12). In an EMA study, participants are usually prompted several times during the day or are asked to self-initiate a report when an event occurs. This largely reduces the risk of retrospective recall bias associated with summative judgements or recollection of behavior patterns [61]. EMA performed on smartphones has a number of advantages including automatic recording of the time stamp of answers in near-real-time, possibility of tracking of compliance and response patterns, and possibility of combining survey responses with other data such as those from a connected monitoring device 
Table 2 Set up of the MRI protocol used in the study

\begin{tabular}{lllll}
\hline MRI sequences and parameters & & & \\
\hline Imaging parameter & 3D T1 MPRAGE & Axial T2 TSE & Axial T2 Dark fluid TSE FS & ep2d diff 3scan trace \\
\hline Repetition time (ms) & 2200 & 3200 & 7910 & 4800 \\
Echo time (ms) & 2.72 & 91 & 83 & 113 \\
Inversion time (ms) & 900 & - & 2359 & - \\
Slice thickness (mm) & 1 & 4 & 4 & 5 \\
Field of view (mm) & 256 & 230 & 215 & 230 \\
Matrix size & $197 \times 256$ & $210 \times 320$ & $179 \times 256$ & $176 \times 176$ \\
Flip angle ( ${ }^{\circ}$ & 8 & 150 & 150 & - \\
Bandwidth (Hz/Px) & 160 & 191 & 190 & 812 \\
Echo train length & - & 7 & 4 & - \\
Signal average & 1 & 2 & 2 & - \\
Number of slices & 192 & 34 & 34 & 20 \\
Acquisition time (min:s) & $3: 39$ & $1: 38$ & $2: 24$ & $1: 23$ \\
\hline
\end{tabular}

MPRAGE (Siemens) Magnetization Prepared - Rapid Gradient Echo; TSE Turbo Spin Echo, FS Fat Saturation

(here the Fitbit Charge 3 monitor) or other data from the smartphone device or from other online sources.

In this study, participants complete four 14-day bursts of EMA data collection. Four pseudo-random surveys (sampling four daily periods: 8-11:59, 12-15:59, 16-19: $59,20: 00-22: 00)$ assess momentary variability in affective states, stress, pain, and context. The morning survey includes questions about sleep and the evening survey (last of the day) also includes some retrospective end-of-day assessments. Additionally, participants are asked to complete two types of self-initiated reports: (1) PA report (episodes of intentional PA lasting at least 20 min and leading to increased heart rate, breathing and sweating) and (2) injury report (when encountering injury associated with physical activity). Every Sunday between 16:00 and 20:00 participants also receive a weekly injury survey (to facilitate prospective monitoring of injury incidence even in cases when a self-initiated injury report may not have been filed). This weekly survey was also enriched by COVID-19 related questions during the pandemic restrictions to evaluate factors associated with PA and mental health (for details please see description at https://www.covidminds.org/longitudinal-studies; The 4HAIE study). Finally, a context-trigged survey is initiated when Fitbit data indicate a decrease in PA in a given week below $90 \%$ confidence interval of the average level of the past 4 weeks. This floating algorithm allows us to detect potential periods of decline in PA that may also be indicative of incurred injury.

In this study, we also collect real-time data about air quality from a network of available public air quality measurement stations. A server application developed for the study specifically aggregates publicly available data on all available air pollution parameters (data downloaded every $30 \mathrm{~min}$ ) and integrates them with
GPS data obtained from the smartphone while protecting participant privacy. Exact GPS location of participants is not recorded, only data from the linked nearest public air quality monitoring station is used each time they make a report of an episode of PA or at least once per day when no activity is reported. Monitoring and combining these different streams of data will allow us to evaluate dynamically the associations among air pollution parameters, behavior and wellbeing, and so gain rich insight into patterns of healthy functioning and within-person fluctuations of variables of interest across moments, days, or weeks. Figure 2 depicts the overview of the entire study protocol including the EMA bursts.

\section{Discussion}

Within this protocol, we introduce a unique interdisciplinary study to longitudinally assess the associations among air pollution, physical (in) activity, runningrelated injuries and psychosocial factors in a sample of $n=1500$ participants across the adult lifespan (18-65 years). As part of the study, rich laboratory assessments are conducted (on physiological, biomechanical, anthropometric, cognitive and neuroimaging variables) in combination with online surveys (sociodemographic, psychological, physical activity, biomechanical variables) to establish baseline characteristics of the study cohort. These cross-sectional assessments will be followed with long-term follow-up assessments 5 years later (as part of a follow-up project) to allow for detection of risk/protective factors in the context of long-term change. More acute, short-term effects of air pollution and PA status on sleep, psychological and injury parameters can also be evaluated due to the dynamic 12-month monitoring (following baseline assessments) using objective sensory data (Fitbit) and mobile apps. These intensive 


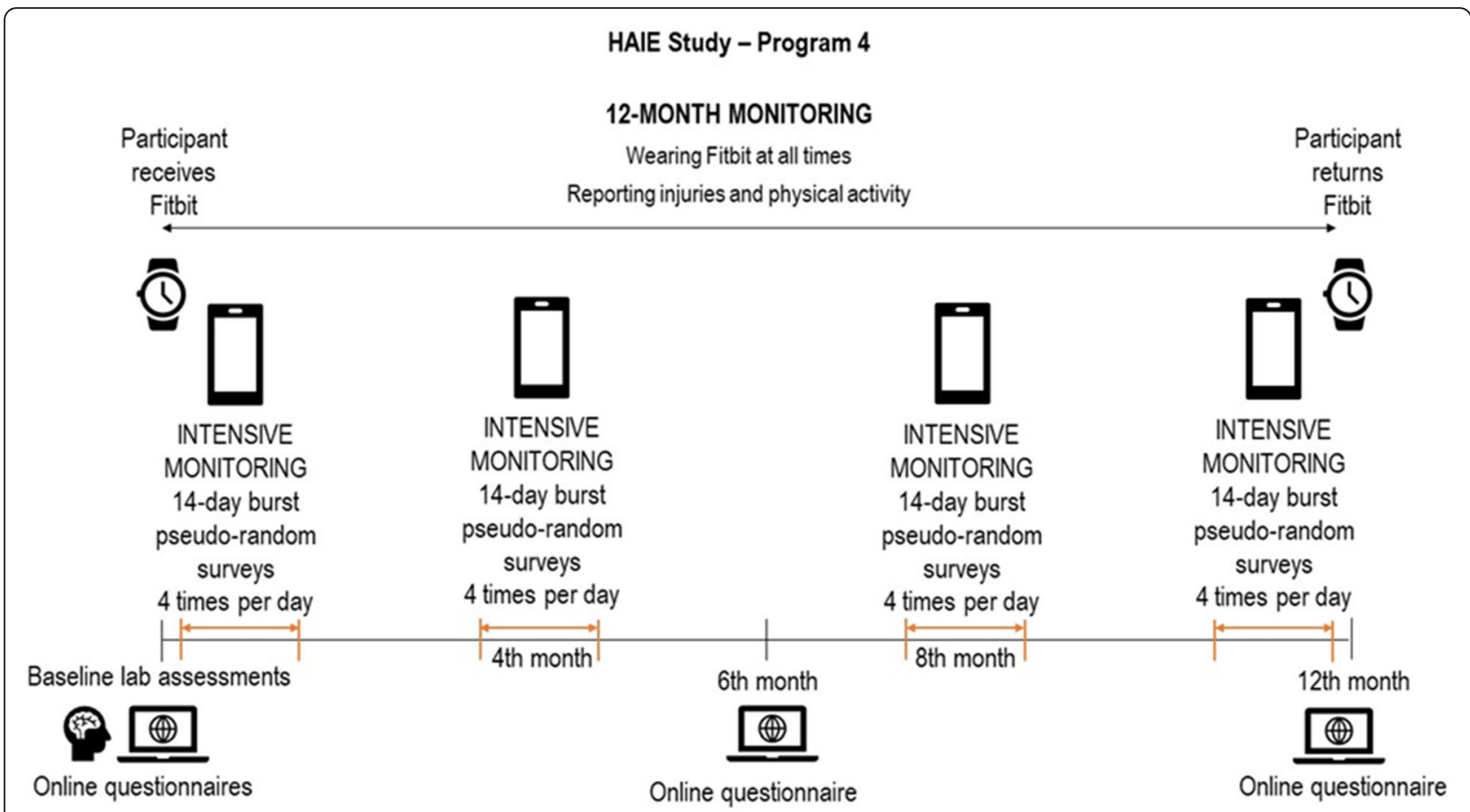

Fig. 2 Graphic overview of the study protocol. Note. Figure adapted with permission from the Czech Information System on Air Quality (ČHMÚ). Data are from 2018 and reflecting limit value valid from 2020

longitudinal data will enable prediction of individual trends in sleep, physical activity, injury risk, and air pollution to help establish suitable future intervention targets.

It is of particular interest in this study to evaluate and detect important predictors of running-related injuries (RRI), including potential differences in incidence rates by air-pollution or PA status. To this end we collect prospective weekly self-report data as well as self-initiated in-time reports of PA-related injuries via the study mobile app. To this date (October 2020), 287 self-initiated reports of occurred injuries have been received from $n=$ 171 participants. In weekly reports, 396 individual reports of injuries have been collected from $n=261$ participants. We have received 7777 at least partially answered PA decrease surveys from $n=844$ participants (additional 1691 surveys were returned unanswered). These data can be aggregated with other EMA and air pollution data to assess whether there are differential rates of injuries across air pollution and PA status and what psychological, biomechanical, or physiological factors may underlie the differences. There is a lack of RRI studies with dynamic EMA psychosocial data in the literature. Some studies suggest that psychosocial variables can be predictors for RRI. For example, scores on self-reported mental health and negative affect were predictive of injury status in another two-year prospective study, with runners who suffered injury reporting worse mental health status and more negative emotions [82]. In addition, dynamic EMA data from HAIE study may indicate whether variations in psychosocial variables may be serve as meaningful predictors of RRI. This information may aid in future interventions aimed at maintaining recommended levels of PA while minimizing runningrelated injuries.

Additionally, longitudinal Fitbit data combined with EMA survey data on the context of PA will allow us to evaluate PA decreases throughout the 12-month monitoring period, including decreases unrelated to injuries. While previous studies have focused on predicting the participation and levels of PA [83, 84], little attention has been devoted to studying for instance weekly or monthly fluctuations and decreases in PA. Although some level of PA is better than none and even dose lower than recommended may bring some health-related benefits [85], it is regular and sustained PA that is likely to garner the most positive effects on health and related variables. It is thus important to evaluate the predictors of decreases in PA and fluctuations specifically, to aid future interventions focused on the long-term maintenance of PA.

The integration of mental and neurocognitive assessments and brain MRI in our study, in combination with data on physiological and behavioural variables, may provide relevant knowledge about the association between air pollution and cognitive and brain health 
outcomes observed in many but not all studies [40, 42$46,48,51,80]$. While PA has been long considered a protective factor for dementia, air pollution has been acknowledged as one of the key risk factors for dementia only recently [86]. The interactions between level of PA and air quality have been therefore rather underinvestigated, supporting the importance of our study. Our findings may contribute to the small amount of literature examining the psychological and neurological benefits of regular exercise in non-polluted and polluted environments [57]. Furthermore, due to wide range of PA levels (particularly in the active group), the 4HAIE study may help to unveil whether the potential association between level of PA and mental and cognitive functioning is linear or not. There might be an optimal level of PA beneficial for the brain while extreme levels of PA might be rather ineffective or even detrimental to brain health.

The interdisciplinary approach of our study and multiple laboratory data collection, including blood markers, cardiac autonomic functioning, and brain MRI imaging, will enable us to investigate potential biological mechanisms linking air-pollution and PA status, to mental and cognitive health. We expect cognitive function and specific brain structures such as prefrontal cortex, white matter and hippocampus, to be lower and smaller in volume, respectively, in adults from air-polluted environment and in inactive controls. Our primary plausible physiological mechanisms underlying the association between air-pollution and worse mental and cognitive health are higher level of chronic inflammation and oxidative stress, lower level of BDNF, and cardiac and vascular functioning [52].

In a longitudinal perspective, based on laboratory data collected in future waves, this study may help to decribe psychological and behavioral factors affecting resilience against age-related changes in the brain, cognition or mental health. This hopefully will generate ways of protecting healthy cognitive and mental aging in airpolluted environment, an approach that is needed, given the current limited progress specifically in preventing dementia [86] and the large number of people chronically exposed to poor air quality [6].

\section{Anticipated challenges and study limitations}

The timing of the study coincided with the COVID-19 pandemic which has presented a unique set of challenges with respect to the main study aims, but also some interesting opportunities. Whereas there is evidence that there have been intermittent reductions in air pollution in response to restrictions on mobility [87], air pollution has been linked with worse COVID-19 disease outcomes $[7,8]$. The pandemic restrictive measures have also led many countries to take drastic restrictions to prevent the transmission of the virus such as limiting human interaction, quarantines, imposing a curfew and even locking down entire cities [88]. While this means that our study will uexpectedly allow us to naturally capture changes in behavior and psychological factors before, during, and after restrictive measures employed in the Czech Republic to combat the pandemic, it poses dilemmas in interpreting the study results. A number of reports have described how the pandemic-related restrictions impacted negatively population mental health [89] and at the same time, the imposed lockdowns have led to improved air quality, albeit intermittent $[87,90]$. In our study, more specifically, in $n=557$ participants at least one EMA burst but no more than three out of the four bursts occurred prior to the first set of restrictions, which came into force on March 12th, 2020. Out of the $\mathrm{n}=557$ participants, 175 participants had completed one EMA burst prior to the restrictions (29 out of these were undergoing their 2nd burst on the day of the first restrictions), $n=225$ participants had completed two bursts (24 of these were undergoing their 3rd burst at that time), and $n=157$ had completed three bursts. Thanks to the 12-month duration and ongoing enrollment of participants, the study is likely to continue past the end of restrictions of the second wave of the pandemic (Fall-Winter 2020), allowing us to capture the impact of variously strict restrictions as well as the return to the normal or "new normal" state of functioning.

The role of PA as a health-enhancing activity has been highlighted also as part of the ongoing COVID-19 pandemic, although ironically opportunities for PA have been reduced as a consequence of pandemic restrictions. Studies indicate that people who reported decreases in PA during COVID-19 mitigation strategies reported increased stress and anxiety levels [91-94]. For some groups of the population, the social distancing and isolation regulations during the pandemic may have put new barriers in engaging in PA [94]. Given that PA represents a potential coping strategy for mental health issues, our study may help shed light on how PA behavior has changed during the COVID-19 pandemic and what factors help predict sustained PA patterns.

The large sample size is a strength of this study. The recruitment strategy involves quota sampling to ensure predetermined distribution by age, gender, air pollution and PA status, still it relies on volunteers who own smartphones and have online access. These requirements precluded drawing a nationally representative sample. For example, based on descriptive data on the first two thirds of participants involved the 4HAIE study, there is about $45 \%$ of men and women with university degree while the average proportion of people with completed university degree is around $24 \%$ in the Czech Republic [95]. Given the fact that education is one of the greatest protective factors in mental and brain health, 
the potential association between air-pollution and mental and cognitive health outcomes and neural correlates may be underestimated due to the higher proportion of people from higher socioeconomic status in our study. Nevertheless, efforts are being made to recruit across social status and target individuals with lower education and income status, and, importantly, to ensure the distribution of the educational level in the 4HAIE cohort is similar across the regions and PA status to minimize impact of selection bias.

Given the interdisciplinary nature of the study, the most intriguing study hypotheses will lie in evaluating interactions among variables at different levels of analysis and across disciplines. Although having laboratory tests means we do not have to rely on self-reported information only, it led to rather extensive assessment batteries that pose high burden on participants. While dropout rates remain low and adherence statistics appear acceptable, future follow-up assessments are likely to be trimmed and streamlined. The large-scale nature of the study is ambitious especially with respect to the EMA data collection which relies on participants' own devices. Although extensive preliminary work preceded deployment of our mobile app and server, ongoing technical issues persist for certain types of devices. This may contribute to the challenge of maintaining optimal response rates in the EMA part of the study and missing data management. The participants receive payment for their baseline laboratory assessments and are eligible to win in a monthly lottery, nonetheless, there are no other explicit incentives to continue within the study and complete the EMA and weekly surveys. With 661 participants past the 6-month study mark, the average completion rate for the momentary EMA surveys is around $70 \%$ with response rates for weekly surveys around $76 \%$. When aiming to maximize compliance in EMA studies, a fine balance must be struck between providing sufficiently motivating extrinsic incentives (e.g., renumeration) and biasing data with too high of an incentive [96]. Intrinsic incentives offer an alternative approach and can be incorporated into existing technologies with advanced features such as elements of gaming or provision of feedback on response rates within the study [97-99]. In the 4HAIE study, we employ ongoing surveillance of study adherence with a system of both automatic notifications and reminders (app) as well as telephone, SMS, and email check-ins and follow-up contacts when necessary. Our approach was designed to align with recommended strategies for optimizing response rates in EMA studies such as incorporating ongoing compliance monitoring, check-in strategies and providing compliancebased incentives to support participant engagement $[99,100]$.

\section{Conclusion}

The 4HAIE study is an interdisciplinary investigation of both long-term and short-term (acute) associations among air pollution, physical (in) activity, sleep, running-related injuries and psychosocial factors across the adult lifespan. Rich laboratory assessments are combined with online surveys and dynamic 12-month monitoring. The study cohort will be followed prospectively (in 5 years) beyond this 12-month period, allowing for the assessment of individual risk factors as well as longterm patterns in physical activity, sleep, injury risk, and air pollution to help establish suitable future intervention targets. Future results of this large-scale study may also serve to underscore the importance of air pollution prevention and mitigation measures to policymakers in the Czech Republic (especially in the highly polluted region).

\section{Abbreviations}

HAIE: Health Aging in Industrial Environment; 4HAIE: Health Aging in Industrial Environment - Program 4; WHO: World Health Organisation; $\mathrm{PM}_{10}$ $\mathrm{PM}_{2.5}$ : Particulate matter; $\mathrm{NO}_{2}$ : Nitrogen dioxide; $\mathrm{CO}$ : Carbon monoxide; $\mathrm{SO}_{2}$ : Sulphur dioxide; AQI: Air quality index; PA: Physical activity; MSR: Moravian-Silesian Region; SBR: South Bohemian Region; MRI: Magnetic resonance imaging; CNS: Central nervous systém; HR: Hazard ration; BDNF: Brain-derived neurotrophic factor; DXA: Dual-energy xray absorptiometry; DREAM: Dynamic Real-time Ecological Ambulatory Methodologies; EMA: Ecological momentary assessment; RRI: Running-related injuries

\section{Acknowledgements}

We would like to thank all students, research assistants and technicians involved in the long-term and challenging data collection during the Covid19 pandemic.

\section{Authors' contributions}

Conceptualization, S.E., V.J., D.J.; methodology, S.E., V.J., D.J. (V.D., T.B., K.J. on neuroimaging; J.K., R.C. on air pollution parameter data collection); data collection, L.K., V.V., M.S., B.K., D.B., J.K., R.C.; writing-original draft preparation, S.E., V.J., L.K., V.V., M.S., B.K.; writing--review and editing, D.J., D.B., V.D., J.K., R.C., T.B., K.J.; supervision, S.E., V.J., D.J.; funding acquisition, D.J. All authors have read and agreed to the published version of the manuscript. All authors read and approved the final manuscript.

\section{Funding}

This publication was based on the information (data) provided from the Program 4 within the research project designated (named) "Healthy Aging in Industrial Environment HAIE" with registration number of CZ.02.1.01/0.0/0.0/ 16_019/0000798, which was funded by the European Union and provided by the Ministry of Education, Youth and Sports of the Czech Republic. The mentioned information (data) was obtained (gathered) by the Department of Human Movement Studies, The Human Motion Diagnostic Center, University of Ostrava, Ostrava, Czech Republic.

\section{Availability of data and materials}

Not applicable.

Ethics approval and consent to participate

Study participants completed a written informed consent. The study procedures were approved by the Ethics Comittee of the Unversity of Ostrava, Czech Republic.

\section{Consent for publication}

Not applicable.

\section{Competing interests}

The authors declare that they have no competing interests.

\section{Author details}

${ }^{1}$ Faculty of Education, University of Ostrava, Ostrava, Czech Republic. ${ }^{2}$ Faculty of Informatics, Masaryk University, Brno, Czech Republic. ${ }^{3}$ Faculty of Medicine, 
University of Ostrava, Ostrava, Czech Republic. ${ }^{4}$ Faculty of Life Sciences, University of Hradec Kralove, Hradec Králové, Czech Republic. ${ }^{5}$ VSB-Technical University of Ostrava, Ostrava, Czech Republic. ${ }^{6}$ University Hospital of Child and Adolescent Psychiatry and Psychotherapy, University of Bern, Bern, Switzerland. ${ }^{7}$ Section for Experimental Child and Adolescent Psychiatry, Department of Child and Adolescent Psychiatry, Centre for Psychosocial Medicine, University of Heidelberg, Heidelberg, Germany.

Received: 1 December 2020 Accepted: 4 January 2021 Published online: 12 January 2021

\section{References}

1. Nations U, of Economic D, Affairs S, Division P. World population ageing 2019: highlights. 2019. https://www.un.org/en/development/desa/ population/publications/pdf/ageing/WorldPopulationAgeing2019-Highlights. pdf. Accessed 25 May 2020.

2. World Health Organization. Air quality guidelines. Global update 2005. Particulate matter, ozone, nitrogen dioxide and sulfur dioxide: World Health Organization; 2017. http://www.euro.who.int/_data/assets/pdf_file/0005/ 78638/E90038.pdf?ua=1. Accessed 25 May 2020.

3. World Health Organization. WHO Air quality guidelines for particulate matter, ozone, nitrogen dioxide and sulfur dioxide: global update 2005. 2005.

4. Kampa M, Castanas E. Human health effects of air pollution. Environ Pollut 2008;151:362-7.

5. Agency EE. Air quality in Europe: 2019 report. 2019. https://www.eea.europa. eu/publications/air-quality-in-europe-2019. Accessed 25 May 2020.

6. World Health Organization. Ambient (outdoor) air pollution. Ambient (outdoor) air pollution. 2018. https://www.who.int/en/news-room/factsheets/detail/ambient-(outdoor)-air-quality-and-health. Accessed 25 May 2020.

7. Ogen Y. Assessing nitrogen dioxide (NO2) levels as a contributing factor to coronavirus (COVID-19) fatality. Sci Total Environ. 2020;726:138605. https:// doi.org/10.1016/j.scitotenv.2020.138605.

8. Zhu Y, Xie J, Huang F, Cao L. Association between short-term exposure to air pollution and COVID-19 infection: evidence from China. Sci Total Environ. 2020;727. https://doi.org/10.1016/j.scitotenv.2020.138704.

9. Raaschou-Nielsen O, Andersen ZJ, Beelen R, Samoli E, Stafoggia M, Weinmayr $\mathrm{G}$, et al. Air pollution and lung cancer incidence in 17 European cohorts: prospective analyses from the European study of cohorts for air pollution effects (ESCAPE). Lancet Oncol. 2013;14:813-22.

10. Cowie H, Crawford J, Davis A, Steinle S, Reis S, Dixon K, et al. Air quality, health, wellbeing and behaviour. 2015.

11. Giles LV, Koehle MS. The health effects of exercising in air pollution. Sports Med. 2014:44:223-49. https://doi.org/10.1007/s40279-013-0108-z.

12. Pasqua LA, Damasceno MV, Cruz R, Matsuda M, Martins MG, Lima-Silva AE, et al. Exercising in air pollution: the cleanest versus dirtiest cities challenge. Int J Environ Res Public Health. 2018;15(7):1502.

13. Slezakova K, Pereira MC, Morais S. Ultrafine particles: levels in ambient air during outdoor sport activities. Environ Pollut. 2020;258:113648.

14. An R, Zhang S, Ji M, Guan C. Impact of ambient air pollution on physical activity among adults: a systematic review and meta-analysis. Perspect Public Health. 2018;138:111-21.

15. An $R$, Xiang $X$. Ambient fine particulate matter air pollution and leisure-time physical inactivity among US adults. Public Health. 2015;129:1637-44.

16. Hu L, Zhu L, Xu Y, Lyu J, Imm K, Yang L. Relationship between air quality and outdoor exercise behavior in China: a novel mobile-based study. Int J Behav Med. 2017;24:520-7.

17. Roberts JD, Voss JD, Knight B. The association of ambient air pollution and physical inactivity in the United States. PLoS One. 2014;9(3):e90143.

18. Yu H, Cheng J, Gordon SP, An R, Yu M, Chen X, et al. Impact of air pollution on sedentary behavior: a cohort study of freshmen at a university in Beijing, China. Int J Environ Res Public Health. 2018;15. https://doi.org/10.3390/ ijerph15122811.

19. Lawrence WR, Yang M, Zhang C, Liu RQ, Lin S, Wang SQ, et al. Association between long-term exposure to air pollution and sleep disorder in Chinese children: the Seven Northeastern Cities study. Sleep. 2018;41(9):Zsy122.

20. Yu H, Chen P, Paige Gordon S, Yu M, Wang Y. The association between air pollution and sleep duration: a cohort study of freshmen at a University in Beijing, China. Int J Environ Res Public Health. 2019;16. https://doi.org/10. 3390/ijerph16183362.
21. Bloemsma LD, Wijga AH, Klompmaker JO, Janssen NAH, Smit HA, Koppelman $\mathrm{GH}$, et al. The associations of air pollution, traffic noise and green space with overweight throughout childhood: the PIAMA birth cohort study. Environ Res. 2019:169:348-56.

22. Bloemsma LD, Gehring U, Klompmaker JO, Hoek G, Janssen NAH, Lebret E, et al. Green space, air pollution, traffic noise and cardiometabolic health in adolescents: the PIAMA birth cohort. Environ Int. 2019;131:104991.

23. del Pozo CB, Alfonso-Rosa RM, McGregor D, Chastin SF, Palarea-Albaladejo J, del Pozo Cruz J. Sedentary behaviour is associated with depression symptoms: compositional data analysis from a representative sample of 3233 US adults and older adults assessed with accelerometers. J Affect Disord. 2020;265:59-62.

24. Costa LG, Cole TB, Dao K, Chang YC, Coburn J, Garrick JM. Effects of air pollution on the nervous system and its possible role in neurodevelopmental and neurodegenerative disorders. Pharmacol Ther. 2020;210:107523.

25. Braithwaite I, Zhang S, Kirkbride JB, Osborn DPJ, Hayes JF. Air pollution (particulate matter) exposure and associations with depression, anxiety, bipolar, psychosis and suicide risk: a systematic review and meta-analysis. Environ Health Perspect. 2019:127. https://doi.org/10.1289/EHP4595.

26. Newbury JB, Arseneault L, Beevers S, Kitwiroon N, Roberts S, Pariante CM, et al. Association of air pollution exposure with psychotic experiences during adolescence. JAMA Psychiatry. 2019;76:614-23. https://doi.org/10. 1001/jamapsychiatry.2019.0056.

27. Petkus AJ, Younan D, Wang X, Serre M, Vizuete W, Resnick S, et al. Particulate air pollutants and trajectories of depressive symptoms in older women. Am J Geriatr Psychiatry. 2019;27:1083-96.

28. Pun VC, Manjourides J, Suh H. Association of ambient air pollution with depressive and anxiety symptoms in older adults: results from the NSHAP study. Environ Health Perspect. 2017;125:342-8.

29. Lim YH, Kim H, Kim JH, Bae S, Park HY, Hong YC. Air pollution and symptoms of depression in elderly adults. Environ Health Perspect. 2012; 120:1023-8.

30. Sass V, Kravitz-Wirtz N, Karceski SM, Hajat A, Crowder K, Takeuchi D. The effects of air pollution on individual psychological distress. Health Place. 2017:48:72-9.

31. Zijlema WL, Wolf $\mathrm{K}$, Emeny $\mathrm{R}$, Ladwig KH, Peters A, Kongsgård $\mathrm{H}$, et al. The association of air pollution and depressed mood in 70,928 individuals from four European cohorts. Int J Hyg Environ Health. 2016;219:212-9.

32. Kawada T. Air pollution and depressed mood: consistency of association. Int J Hyg Environ Health. 2016;219:477. https://doi.org/10.1016/j.jjheh.2016.01.003.

33. Zijlema WL, Stolk RP, Rosmalen JGM. Response to "air pollution and depressed mood: consistency of association. Air pollution and cardiovascular mortality: caution for meta-analysis". Int J Hyg Environ Health. 2016;219:478-9. https://doi.org/10.1016/j.ijheh.2016.02.005.

34. Gu D, Huang N, Zhang M, Wang F. Under the dome: air pollution, wellbeing, and pro-environmental behaviour among Beijing residents. J Pacific Rim Psychol. 2015;9:65-77.

35. Perach R, Allen CK, Kapantai I, Madrid-Valero JJ, Miles E, Charlton RA, et al. The psychological wellbeing outcomes of nonpharmacological interventions for older persons with insomnia symptoms: a systematic review and meta-analysis. Sleep Med Rev. 2019:43:1-13.

36. Murawski B, Wade L, Plotnikoff RC, Lubans DR, Duncan MJ. A systematic review and meta-analysis of cognitive and behavioral interventions to improve sleep health in adults without sleep disorders. Sleep Med Rev. 2018;40:160-9.

37. Zhai L, Zhang H, Zhang D. Sleep duration and depression among adults: a meta-analysis of prospective studies. Depress Anxiety. 2015;32:664-70.

38. McDowell CP, Dishman RK, Gordon BR, Herring MP. Physical activity and anxiety: a systematic review and meta-analysis of prospective cohort studies. Am J Prev Med. 2019:57:545-56.

39. Rebar AL, Stanton R, Geard D, Short C, Duncan MJ, Vandelanotte C. A metameta-analysis of the effect of physical activity on depression and anxiety in non-clinical adult populations. Health Psychol Rev. 2015;9:366-78.

40. Clifford A, Lang L, Chen R, Anstey KJ, Seaton A. Exposure to air pollution and cognitive functioning across the life course - a systematic literature review. Environ Res. 2016;147:383-98.

41. Underwood E. Brain pollution: evidence builds that dirty air causes Alzheimer's, dementia. Science. 2017;355(6323):342-5. https://doi.org/10. 1126/science.355.6323.342.

42. Chen H, Kwong JC, Copes R, Tu K, Villeneuve PJ, van Donkelaar A, et al. Living near major roads and the incidence of dementia, Parkinson's disease, 
and multiple sclerosis: a population-based cohort study. Lancet. 2017;389: 718-26. https://doi.org/10.1016/S0140-6736(16)32399-6.

43. Kilian J, Kitazawa M. The emerging risk of exposure to air pollution on cognitive decline and Alzheimer's disease - evidence from epidemiological and animal studies. Biom J. 2018;41:141-62.

44. Russ TC, Reis S, Van Tongeren M. Air pollution and brain health: defining the research agenda. Curr Opin Psychiatry. 2019;32:97-104. https://doi.org/ 10.1097/YCO.0000000000000480.

45. Younan D, Petkus AJ, Widaman KF, Wang X, Casanova R, Espeland MA, et al. Particulate matter and episodic memory decline mediated by early neuroanatomic biomarkers of Alzheimer's disease. Brain. 2020;143:289-302. https://doi.org/10.1093/brain/awz348.

46. de Prado BP, Mercader EMH, Pujol J, Sunyer J, Mortamais M. The effects of air pollution on the brain: a review of studies interfacing environmental epidemiology and neuroimaging. Curr Environ Health Rep. 2018:5:351-64. https://doi.org/10.1007/s40572-018-0209-9.

47. Hahad O, Lelieveld J, Birklein F, Lieb K, Daiber A, Münzel T. Ambient air pollution increases the risk of cerebrovascular and neuropsychiatric disorders through induction of inflammation and oxidative stress. Int J Mol Sci. 2020;21:1-24. https://doi.org/10.3390/ijms21124306.

48. Kulick ER, Wellenius GA, Boehme AK, Joyce NR, Schupf N, Kaufman JD, et al. Long-term exposure to air pollution and trajectories of cognitive decline among older adults. Neurology. 2020;94:E1782-92. https://doi.org/10.1212/ WNL.0000000000009314.

49. Gatto NM, Henderson WW, Hodis HN, St. John JA, Lurmann F, Chen JC, et al. Components of air pollution and cognitive function in middle-aged and older adults in Los Angeles. Neurotoxicology. 2014;40:1-7.

50. Weuve J, Puett RC, Schwartz J, Yanosky JD, Laden F, Grodstein F. Exposure to particulate air pollution and cognitive decline in older women. Arch Intern Med. 2012;172:219-27.

51. Cserbik D, Chen JC, McConnell R, Berhane K, Sowell ER, Schwartz J, et al. Fine particulate matter exposure during childhood relates to hemisphericspecific differences in brain structure. Environ Int. 2020;143. https://doi.org/ 10.1016/j.envint.2020.105933.

52. Allen JL, Klocke C, Morris-Schaffer K, Conrad K, Sobolewski M, Cory-Slechta DA. Cognitive effects of air pollution exposures and potential mechanistic underpinnings. Curr Environ Health Rep. 2017:4:180-91.

53. Hamer M, Chida Y. Physical activity and risk of neurodegenerative disease: a systematic review of prospective evidence. Psychol Med. 2009;39-42. https://doi.org/10.1017/S0033291708003681.

54. Erickson Kl, Hillman C, Stillman CM, Ballard RM, Bloodgood B, Conroy DE, et al. Physical activity, cognition, and brain outcomes: a review of the 2018 physical activity guidelines. Med Sci Sports Exerc. 2019;51:1242-51.

55. Vaynman S, Ying Z, Gomez-Pinilla F. Hippocampal BDNF mediates the efficacy of exercise on synaptic plasticity and cognition. Eur J Neurosci. 2004;20:2580-90. https://doi.org/10.1111/j.1460-9568.2004.03720.x.

56. Cipriani G, Danti S, Carlesi C, Borin G. Danger in the air: air pollution and cognitive dysfunction. Am J Alzheimers Dis Other Dement. 2018;33:333-41.

57. Bos I, De Boever P, Int Panis L, Meeusen R. Physical activity, air pollution and the brain. Sports Med. 2014;44:1505-18. https://doi.org/10.1007/s40279-0140222-6.

58. Buoli M, Grassi S, Caldiroli A, Carnevali GS, Mucci F, lodice S, et al. Is there a link between air pollution and mental disorders? Environ Int. 2018;118:154-68.

59. Altuğ H, Fuks KB, Hüls A, Mayer A-K, Tham R, Krutmann J, et al. Air pollution is associated with depressive symptoms in elderly women with cognitive impairment. Environ Int. 2020;136:105448. https:/doi.org/10.1016/j.envint2019.105448.

60. Center PR. Smartphone ownership and internet usage continues to climb in emerging economies. 2016. http://www.pewglobal.org/2016/02/22/ smartphone-ownership-and-internet-usage-continues-to-climb-in-emergingeconomies/.

61. Stone A, Shiffman S, Atienza A, Nebeling L. The science of real-time data capture: self-reports in health research. New York: Oxford University Press; 2007.

62. Office CS. Life tables for the Czech Republic, cohesions regions, and regions. https://www.czso.cz/csu/czso/life-tables-for-the-czech-republic-cohesionregions-and-regions-2017-2018. Accessed 1 Oct 2020.

63. Hůnová I. Ambient air quality in the Czech Republic: past and present. Atmosphere (Basel). 2020;11:0-26.

64. Sram RJ, Binkova B, Dostal M, Merkerova-Dostalova M, Libalova H, Milcova A, et al. Health impact of air pollution to children. Int J Hyg Environ Health. 2013;216:533-40.
65. Fact Sheet Physical Activity Global recommendations on physical activity for health Consequences of physical inactivity. www.euro.who.int/physicalactivity. Accessed 4 Nov 2020.

66. Staufenbiel SM, Penninx BWJH, Spijker AT, Elzinga BM, van Rossum EFC. Hair cortisol, stress exposure, and mental health in humans: a systematic review. Psychoneuroendocrinology. 2013;38:1220-35.

67. Sapolsky RM. Glucocorticoids and hippocampal atrophy in neuropsychiatric disorders; 2000.

68. Stalder T, Steudte-Schmiedgen S, Alexander N, Klucken T, Vater A, Wichmann S, et al. Stress-related and basic determinants of hair cortisol in humans: a meta-analysis. Psychoneuroendocrinology. 2017;77:261-74

69. Manenschijn L, Koper JW, Lamberts SWJ, Van Rossum EFC. Evaluation of a method to measure long term cortisol levels. Steroids. 2011;76:1032-6.

70. Cooper GAA, Kronstrand R, Kintz P. Society of hair testing guidelines for drug testing in hair. Forensic Sci Int. 2012;218:20-4.

71. Wennig R. Potential problems with the interpretation of hair analysis results. 2000. www.elsevier.com/locate/forsciint.

72. Gonzalez D, Jacobsen D, Ibar C, Pavan C, Monti J, Fernandez Machulsky N, et al. Hair cortisol measurement by an automated method. Sci Rep. 2019; 9(1):1-6.

73. Russell E, Kirschbaum C, Laudenslager ML, Stalder T, De Rijke Y, Van Rossum EFC, et al. Toward standardization of hair cortisol measurement: results of the first international interlaboratory round robin. 2014

74. Rey A. L'examen clinique en psychologie (The Clinical Psychological Examination). Paris: Presse Universitaires de France; 1964.

75. Schoenberg MR, Dawson KA, Duff K, Patton D, Scott JG, Adams RL. Test performance and classification statistics for the Rey Auditory Verbal Learning Test in selected clinical samples. Arch Clin Neuropsychol. 2006;21: 693-703. https://doi.org/10.1016/j.acn.2006.06.010.

76. Preiss $\mathrm{M}$, Bartoš $\mathrm{A}$, Čermáková $\mathrm{R}$, Nondek $\mathrm{M}$, Benešová $\mathrm{M}$, Rodriguez $\mathrm{M}$, et al. Neuropsychologická baterie Psychiatrického centra Praha, Klinické vyšetření základních kognitivních funkcí. 3rd ed. Praha: Psychiatrické centrum Praha; 2012.

77. Mioshi E, Dawson K, Mitchell J, Arnold R, Hodges JR. The Addenbrooke's Cognitive Examination revised (ACE-R): a brief cognitive test battery for dementia screening. Int J Geriatr Psychiatry. 2006;21:1078-85. https://doi. org/10.1002/gps.1610.

78. Kramer JH, Mungas D, Possin KL, Rankin KP, Boxer AL, Rosen HJ, et al. NIH EXAMINER: conceptualization and development of an executive function battery. J Int Neuropsychol Soc. 2014;20:11-9. https://doi.org/10.1017/ S1355617713001094

79. Peirce J, Gray JR, Simpson S, MacAskill M, Höchenberger R, Sogo H, et al. PsychoPyz: experiments in behavior made easy. Behav Res Methods. 2019; 51:195-203. https://doi.org/10.3758/s13428-018-01193-y.

80. Hagler DJ, Hatton SN, Cornejo MD, Makowski C, Fair DA, Dick AS, et al. Image processing and analysis methods for the adolescent brain cognitive development study. Neuroimage. 2019;202. https://doi.org/10.1016/j. neuroimage.2019.116091.

81. Adler, S., Whitaker, K., Semmelroch, M., Wagstyl K. MELD protocol 3 FreeSurfer quality control. 2018. https://www.protocols.io/view/meldprotocol-3-freesurfer-quality-control-pdadi2e. Accessed 21 Nov 2020.

82. Messier SP, Martin DF, Mihalko SL, Ip E, DeVita P, Cannon DW, et al. A 2-year prospective cohort study of overuse running injuries: the runners and injury longitudinal study (TRAlLS). Am J Sports Med. 2018:46:2211-21.

83. Bauman AE, Reis RS, Sallis JF, Wells JC, Loos RJF, Martin BW, et al. Correlates of physical activity: why are some people physically active and others not? Lancet. 2012;380:258-71. https://doi.org/10.1016/S0140-6736(12)60735-1.

84. Choi J, Lee M, Lee JK, Kang D, Choi JY. Correlates associated with participation in physical activity among adults: a systematic review of reviews and update. BMC Public Health. 2017;17. https://doi.org/10.1186/ s12889-017-4255-2.

85. Geidl W, Schlesinger S, Mino E, Miranda L, Pfeifer K. Dose-response relationship between physical activity and mortality in adults with noncommunicable diseases: a systematic review and meta-analysis of prospective observational studies. Int J Behav Nutr Phys Act. 2020;17:109. https://doi.org/10.1186/s12966-020-01007-5.

86. Livingston G, Huntley J, Sommerlad A, Ames D, Ballard C, Banerjee S, et al. Dementia prevention, intervention, and care: 2020 report of the lancet commission. Lancet. 2020;396:413-46. https://doi.org/10.1016/S01406736(20)30367-6. 
87. Kumari P, Toshniwal D. Impact of lockdown on air quality over major cities across the globe during COVID-19 pandemic. Urban Clim. 2020;34:100719. https://doi.org/10.1016/j.uclim.2020.100719.

88. Zajenkowski M, Jonason PK, Leniarska M, Kozakiewicz Z. Who complies with the restrictions to reduce the spread of COVID-19?: personality and perceptions of the COVID-19 situation. Pers Individ Dif. 2020;166:110199. https://doi.org/10.1016/j.paid.2020.110199.

89. Dawel A, Shou Y, Smithson M, Cherbuin N, Banfield M, Calear AL, et al. The effect of COVID-19 on mental health and wellbeing in a representative sample of Australian adults. Front Psychiatry. 2020;11:579985. https://doi. org/10.3389/fpsyt.2020.579985.

90. He G, Pan Y, Tanaka T. Urban air pollution in China. Nat Sustain. 2020.

91. Martinez EZ, Silva FM, Morigi TZ, Zucoloto ML, Silva TL, Joaquim AG, et al. Physical activity in periods of social distancing due to covid-19: a crosssectional survey. Cienc e Saude Coletiva. 2020;25:4157-68.

92. Meyer J, McDowell C, Lansing J, Brower C, Smith L, Tully M, et al. Changes in physical activity and sedentary behavior in response to covid-19 and their associations with mental health in 3052 us adults. Int J Environ Res Public Health. 2020;17:1-13.

93. Dunca GE, Aver AR, Seto E, Tsang S. Perceived change in physical activity levels and mental health during COVID-19: findings among adult twin pairs. PLoS One. 2020;15(8 August):1-12.

94. Faulkner G, Rhodes RE, Vanderloo LM, Chulak-Bozer T, O'Reilly N, Ferguson $\mathrm{L}$, et al. Physical activity as a coping strategy for mental health due to the COVID-19 virus: a potential disconnect among Canadian adults? Front Commun. 2020:5(September):1-5.

95. OECD. Education at a glance 2020: OECD; 2020. https://doi.org/10.1787/ 69096873-en.

96. Napa Scollon C, Prieto C-K, Diener E. Experience sampling: promises and pitfalls, strength and weaknesses. Dordrecht: Springer; 2009. p. 157-80. https://doi.org/10.1007/978-90-481-2354-4_8.

97. Doherty K, Balaskas A, Doherty G. The design of ecological momentary assessment technologies. Interact Comput. 2020;00. https://doi.org/10.1093/ iwcomp/iwaa019.

98. Heron KE, Smyth JM. Ecological momentary interventions: incorporating mobile technology into psychosocial and health behaviour treatments. Br J Health Psychol. 2010;15(Pt 1):1-39. https://doi.org/10.1348/ $135910709 \times 466063$.

99. Heron KE, Everhart RS, McHale SM, Smyth JM. Using mobile-technologybased ecological momentary assessment (EMA) methods with youth: a systematic review and recommendations. J Pediatr Psychol. 2017;42:1087107. https://doi.org/10.1093/jpepsy/jsx078.

100. Degroote L, Desmet A, De Bourdeaudhuij I, Van Dyck D, Crombez G. Content validity and methodological considerations in ecological momentary assessment studies on physical activity and sedentary behaviour: a systematic review. Int I Behav Nutr Phys Act. 2020;17:35. https://doi.org/10.1186/s12966-020-00932-9.

\section{Publisher's Note}

Springer Nature remains neutral with regard to jurisdictional claims in published maps and institutional affiliations.

Ready to submit your research? Choose BMC and benefit from:

- fast, convenient online submission

- thorough peer review by experienced researchers in your field

- rapid publication on acceptance

- support for research data, including large and complex data types

- gold Open Access which fosters wider collaboration and increased citations

- maximum visibility for your research: over $100 \mathrm{M}$ website views per year

At $\mathrm{BMC}$, research is always in progress.

Learn more biomedcentral.com/submissions 